\title{
Arte colaborativo e identidad en la formación inicial del profesorado
}

\author{
María Dolores Álvarez Rodríguez y Alice Bajardi. Universidad de Granada
}

Recepción: 12 de abril de 2015 | Revisión: 30 de mayo de 2015 | Aceptación/Publicación: 24 de julio de 2015

Correspondencia: alvarezr@ugr.es | alicebajardi@ugr.es | http://hdl.handle.net/10481/37125

Resumen: En este texto presentamos nuestra experiencia en la formación inicial del profesorado de Educación Primaria desarrollando un programa basado en la prácticas artísticas colaborativas y el aprendizaje cooperativo. La estructura actual de la enseñanza universitaria en nuestro entorno está condicionada por los cambios recientes acaecidos en su adaptación al Espacio Europeo de Educación Superior. Se establece que parte de la docencia se imparta en grupos medianos y pequeños. Esta manera de estructurar los grupos para el trabajo docente y para el aprendizaje nos ha llevado a diseñar e implementar una serie de actividades que, en nuestro caso, hemos dotado de un carácter colaborativo y cooperativo. Pensamos que estas prácticas son especialmente interesantes e importantes para la formación artística y pedagógica de los futuros maestros. Presentamos nuestra experiencia teniendo en cuenta los resultados obtenidos en función de nuestros objetivos y contexto.

Palabras Clave: Identidad profesional | Identidad personal | Educación artística | Producción colaborativa | Aprendizaje cooperativo | Formación inicial de profesorado | Competencias docente

\section{COLLABORATIVE ART AND IDENTITY IN PRE-SERVICE TEACHER TRAINING}

\begin{abstract}
In this paper we present our experience in pre-service teacher training for Primary Education based on the development of collaborative art practices and a cooperative learning program. The current structure of university degrees, in the frame of the European Higher Education Area, provides that part of teaching is organized in medium and small groups. This structure of the groups for teaching and learning has enabled us to design and implement a series of activities with collaborative and cooperative nature. We think that such practices are particularly interesting and important for artistic and pedagogical training of future teachers. We present our experience taking into account the results we have obtained following our objectives and context.
\end{abstract}

Keywords: Professional Identity | Personal Identity | Art Education | Collaborative Production | Cooperative Learning | Pre-service Teacher Training | Teaching Competencies

\section{Introducción}

En la tarea docente es necesario reflexionar sobre las prácticas que se realizan y repensar sobre lo ya estipulado. Esta premisa básica es importantante tanto en contextos educativos formales como no formales: documentar, reflexionar, mejorar, evaluar en definitiva, pero cuando se trata de una meta-docencia, de una docncia para la docencia o de un meta-aprendizaje, de un aprendizaje para producir a su vez un aprendizaje, este hecho se multiplica en importancia porque estamos estableciendo las bases exponenciales sobre unas circunstancias y contextos que nos superarán física y temporalmente. Esto sucede con la formación del profesorado. Por otra parte los curricula para la formación del profesorado han cambiado en los últimos años forzados circustancialmente por la adaptación de la enseñanza superior al Espacio Europeo de Educación Superior. Esto ha supuesto, sigue y debe seguir suponiendo, un esfuerzo enorme de coordinación entre el profesorado y ha propiciado un espacio de reflexión, crítica y debate sobre los contenidos teóricos y prácticos de las programaciones, las actividades, la evaluación, e incluso los objetivos específicos. En este contexto de cambio, intercambio y negociación entre los equipos docentes hemos visto adecuado introducir a los estudiantes, futuros profesores de Educación Primaria, en la prácticas artísticas colaborativas a través de un aprendizaje cooperativo. Pensamos que este tipo de actividades van a contribuir de manera poderosa a su formación como docentes. Mediante el desarrollo de capacidades intrapersonales e interpersonales en relación con las prácticas artísticas ayudaremos a construir su identidad profesional docente, no sólo en relación con la materia de educación artística, sino de una manera más global (Read, 2007; Eisner, 1992; Efland, 2004). 


\section{Contribuciones de las prácticas artísticas colaborativas y del aprendizaje cooperativo a la identidad docente en formación}

El arte colaborativo está asociado a la idea de comunidad. Una comunidad suele estar marcada por unos determinantes espaciales pero también se refiere a las personas que la conforman, a sus expectativas, intereses, necesidades, circunstancia vitales y a las relaciones que se establecen entre ellos. En definitiva una comunidad tiene una cultura compartida. Las prácticas artísticas comunitarias también suelen vincularse con colectivos minoritarios o en riesgo de exclusión social. Habitualmente se suelen realizar en zonas socialmente desfavorecidas $y$, en numerosas ocasiones, tienen un carácter crítico, de denuncia o presentan connotaciones políticas e ideológicas. Sin embargo la actividad artística colaborativa tiene múltiples facetas y realidades y ha cubierto diversos objetivos desde sus orígenes, siempre partiendo de la idea de creación y autoría colectiva, con carácter procesual, y de implicación de determinadas circunstancias socio-culturales. Para nosotros estas tres características son determinantes. Tanto la autoría colectiva como el carácter procesual, hacen que el énfasis se ponga en los participantes y en la elaboración de sus procesos relacionales y creativos, pero todo ello contextualizado en una microcultura concreta con connotaciones sociales. En este caso se trata de la cultura del profesorado de educación primaria en formacion inicial, con una serie de características inherentes a su propia condición y posicionamento con respecto al mundo universitario al que se acaba de incorporar y, por supuesto, a su propia elección profesional y su identidad como futuro docente. Más concretamente, en relación con nuestra materia, hemos de referirnos a sus ideas sobre la educación artística escolar que vienen fuertemente enraizadas por su propia experiencia en los años escolares y por lo que social y culturalmente se entiende por arte escolar. Existen, por tanto, unas características identitarias concretas que incluso podríamos sistematizar fácilmente con un estudio básico de carácterísticas demográficas, expectativas, etc., que no es el objetivo en este caso, pero que sí somos capaces de identificar después de años de experiencia docente en este ámbito. Lo que nos interesa es destacar que existe una cultura específica asociada a este colectivo y que las caracterísica identitarias puede facilmente devenir en estereotipos, no sólo personales sino culturales y sociales. En este contexto las prácticas colaborativas son una aportación de gran interés porque por su propia esencia se entienden como ajenas al sistema institucional del arte pero, también al sistema institucional educativo. De esta forma, al alejarse de lo establecido, también subvierten el estereotipo que están tan enraizado y permiten re-pensar en la identidad docente de una manera más fresca y menos condicionada. El carácter crítico de la actividad artística colaborativa contribuye a la reconstrucción de la identidad docente y a la re-elaboración del imaginario en relación con las artes visuales. Se crea un espacio dialógico de aprendizaje que intenta superar la dicotomía observadorcreador, determinada incluso curricularmente, intercambiando roles y tomando conciencia de las implicaciones sociales de la actividad artística en la escuela que no debe ser un ente aislado.

Por otra parte consideramos que el aprendizaje cooperativo puede ser una de las claves para propiciar el cambio la transformación social. Todo aprendizaje supone un cambio y una tansformación. Su origen está en el planteamiento pedagógico de Dewey (2007), al que tanto debemos en la educación artística, y ha propiciado el desarrollo posterior de las dinámicas de aprendizaje en grupo, la interacción en el aprendizaje y incluso las teorías sobre el trabajo y tareas en grupo (Lobato, 1997). Consideramos que el aprendizaje cooperativo conlleva una serie de características que lo hace especialmente adecuado para la formación del profesorado. Las tareas cooperativas conllevan una interdependencia y co-responsabilidad entre los miembros del grupo para asumir una labor común, desarrollando diversidad de roles que favorecen el aprendizaje. Otro aspecto a considerar es la hetereogeneidad de los 
miembros del grupo que propicia la adquisición de los roles adecuados en la microcultura de la formación incial del profesorado. El carácter relacional y contextual está también implícito en el trabajo cooperativo. Este tipo de aprendizaje confronta a los estudiantes en formación con un tipo de tarea y unas circunstancias que serán comunes en su vida profesional futura. Si el aprendizaje supone una transformación también es una evolución que va a producirse durante toda la vida laboral. Se trata de una identidad profesional, que también está en proceso, y que se re-crea en el momento en que se re-piensa. La evaluación del grupo y de los participantes es inherente al aprendizaje cooperativo y la entendemos como la constante reelaboración de los procesos.

\section{Experiencias y prácticas en la formación inicial del profesorado de la Universidad de Granada}

A continuación presentamos algunas de las prácticas que hemos implementado en las cuales los maestros en formación inicial de la Facultad de Ciencias de la Educación de la Universidad de Granada trabajaron en grupos de unas 4-6 personas desarrollando las actividades del Curso bilingüe "Teaching and Learning of Visual Arts". Los grupos se formaron tratando de crear un cierto grado de diversidad en cuanto a las competencias de sus miembros e intentamos que se mantuvieran durante todo el curso para fomentar la responsabilidad, la coherencia y facilitar el desarrollo de habilidades socio-emocionales, comunicativas y colaborativas, incluso desafiando las dificultades de las diferencias sociales y culturales, que resultaron ser un factor enriquecedor. En particular, la presencia de numerosos estudiantes Erasmus procedentes de siete estados diferentes ha enriquecido enormemente el curso desde el punto de vista lingüístico, y sobre todo, cultural.

Hemos tratado en todo momento de promover un ambiente de relación positiva entre los participantes ya que la idea es sentar las bases del curso precisamente sobre las competencias relacionales y emocionales a partir del trabajo igualmente cooperativo y colaborativo de diseño del curso realizado por las dos docentes que antes, durante y después del curso tuvieron que cubrir las diferentes necesidades de mismo. Entre las habilidades mencionadas nos parece necesario señalar el uso eficaz de la comunicación verbal y no verbal y de las TICs como herramientas integradas de enseñanza, la regulación de las dinámicas de grupo, el manejo de las emociones y de los conflictos y la resolución creativa de problemas. Todas ellas hacen una contribución conjunta y esencial a la identidad profesional docente (Bajardi y ÁlvarezRodríguez, 2012; 2014).

Para iniciar el trabajo de grupo involucramos a los estudiantes en el diseño de mapas mentales y conceptuales; entre los primeros mapas se trató "que es el arte" y "por qué enseñar arte" $y$, a partir de ello, propusimos las primeras presentaciones orales, destacando el tono de la voz, el lenguaje corporal, el control del estrés y de las emociones, competencias comunicativas y relacionales valoradas por los futuros docentes como aspectos profesionales especialmente importantes que deben desarrollarse en formación inicial.

Entre otras, destacamos las prácticas colaborativas e individuales de educación artística que se desarrollaron en el ejercicio "Arqueología del 20.000 d.C." con el objetivo de aprender de nuevo, de re-pensar la cultura material, a partir de la observación de los objetos cotidianos para asignarles nuevos contextos y significados. En relación con esta actividad propusimos la autoevaluación en grupos, aplicando la co-evaluación y la evaluación por pares para un proceso de mejora continua. Además, desarrollamos un proceso de inmersión en los distintos modelos de educación artística 
desde el pasado hasta el presente, experimentando ejercicios académicos de dibujo, de auto-expresión realizados con la música, con la pintura y la escultura y reflexión sobre los estereotipos, el análisis de los elementos de la comunicación visual y los procesos de creación colectiva en comunicación gráfica visual. También, organizamos unas sesiones de trabajo en el Centro José Guerrero y en el Museo Memoria de Andalucía. Igualmente se ha diseñado por grupos en cooperación un proyecto educativo de aplicación en la Educación Primaria.

Como actividad individual hicimos un ejercicio de observación y experimentación frente a un espejo seguido por realización de un autorretrato con técnica mixta sobre papel, y también un autorretrato metafórico a través de objetos personales de uso diario. Se organizó una exposición artística con las obras, textos, y material de índole diversa que se elaboró en el marco de esta actividad y resultó una muestra muy significativa que confrontaba la identidad personal y la identidad docente. Se recabaron opiniones a través de entrevistas y cuestionarios lo que permitió una evaluación continua y una re-elaboración conceptual sobre los resultados. En el transcurso de la exposición se desarrolló una experiencia de intercambio entre el profesorado en formación y un grupo de alumnos de educación secundaria que se inician en la investigación universitaria a través de un proyecto, precisamente, sobre la identidad y el retrato. Este intercambio permitió a los futuros docentes enfrentarse de manera dialógica a la temática de la identidad y enfrentarse de manera concreta con alumnado real.

Figura 1: Alice Bajardi (2015) Portrait Exhibition. Photo Series composed by two photos by the author. Left, (2015) Guess who portrait; right, (2015) Box Portrait.

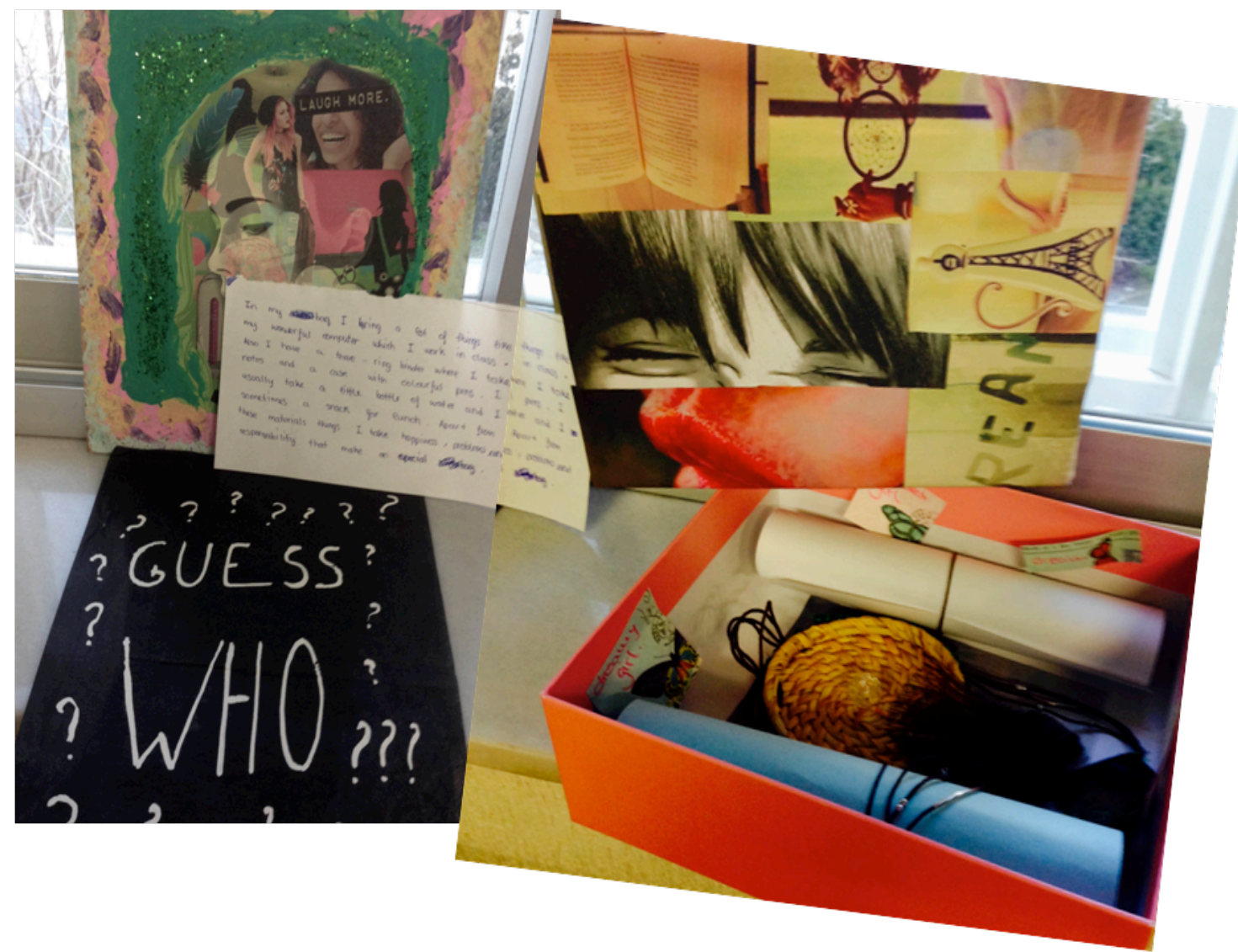

Por otra parte, la evaluación de los estudiantes se llevó a cabo tanto de manera grupal como de forma individual, para responsabilizar a cada miembro de los grupos de los resultados obtenidos a través de las prácticas colectivas, pero también para proporcionar retroalimentación sobre las prácticas individuales. Además, por razones 
logísticas, principalmente relacionadas con los horarios de otros cursos, algunos grupos habían cambiado una parte de sus miembros.

Como resultado del trabajo cooperativo y colaborativo, algunos estudiantes que en principio no eran muy participativos y tenía baja autoestima, cambiaron de actitud que devino, a través de las prácticas del grupo basado en las artes, en posiciones más seguras y participativas. Sin embargo los estudiantes que tendían a imponer y superponer sus propias Ideas sobre las de los otros han demostrado durante las últimas actividades en el aula más empatía hacia los espacios, los tiempos y las ideas de los demás. Además, entre los miembros de cada grupo se produjo un aprendizaje hacia el intercambio de recursos de información y de materiales, estableciendo una comunicación eficaz, compartiendo las cargas para alcanzar los objetivos comunes y los objetivos individuales.

\section{Resultados y conclusiones}

En el cuestionario, proporcionado a los participantes al final de las actividades de seminario, se pidió la opinión de los estudiantes sobre los términos "identidad personal" e "identidad profesional" y sobre sus posibles factores de cambio. Además, se solicitó indicar, motivando la elección, cuál entre todas las actividades de educación artística que experimentamos durante el curso había estimulado especialmente su identidad personal y cuál había desarrollado más su identidad profesional.

Las respuestas que los docentes en formación inicial dieron al susodicho cuestionario fueron muy distintas. Sin embargo, muchas de las respuestas convergieron en indicar cómo actividades especialmente útiles para reflexionar sobre la identidad personal el ejercicio de observación y experimentación frente al espejo, seguido por la realización de un autorretrato con técnica mixta sobre papel, las actividades basadas en el modelo de la educación artística de auto-expresión hechas con la música, seguidas por la reflexión sobre los estereotipos y, por último, la actividad de mayor duración en la que realizaron un autorretrato metafórico a través de objetos personales de uso diario con exposición colectiva final y recogida de opiniones mediante entrevistas (este última se reportó como actividad útil también para desarrollar la identidad profesional).

Con respecto a las actividades que más han desarrollado la identidad profesional de estos futuros educadores se indicaron la compleja actividad de programación de un proyecto educativo para que exigía una colaboración y cooperación mayor si cabe (habilidades para el futuro), y las sesiones de trabajo en los museos (Centro José Guerrero y Museo Memoria de Andalucía) para experimentar una manera diferente de enseñar y aprender sobre arte.

Durante todo el curso se trataron varios argumentos de educación artística incluyendo objetivos, metodologías, instrumentos, habilidades y competencias, y, especialmente durante los seminarios, se mantuvo una constante atención tanto en desarrollar las competencias socio-emocionales, colaborativas y comunicativas, como en estimular el desarrollo de la identidad personal y profesional del profesorado en formación inicial. Sin embargo los términos "identidad personal" y "identidad profesional", aunque ampliamente tratados, en ningún momento se definieron. Se creyó oportuno no asignar un único sentido a estos dos términos sino abrir numerosas posibilidades, propuestas e interrogantes que poderles atribuir y dinámicamente transformar.

En efecto, creemos que la educación artística es un instrumento especialmente útil para el desarrollo de las competencias comunicativas y socio-emocionales, las cooperativas y las colaborativas, y para la búsqueda tanto de la identidad personal 
como de la identidad profesional de una forma dinámica y crítica.

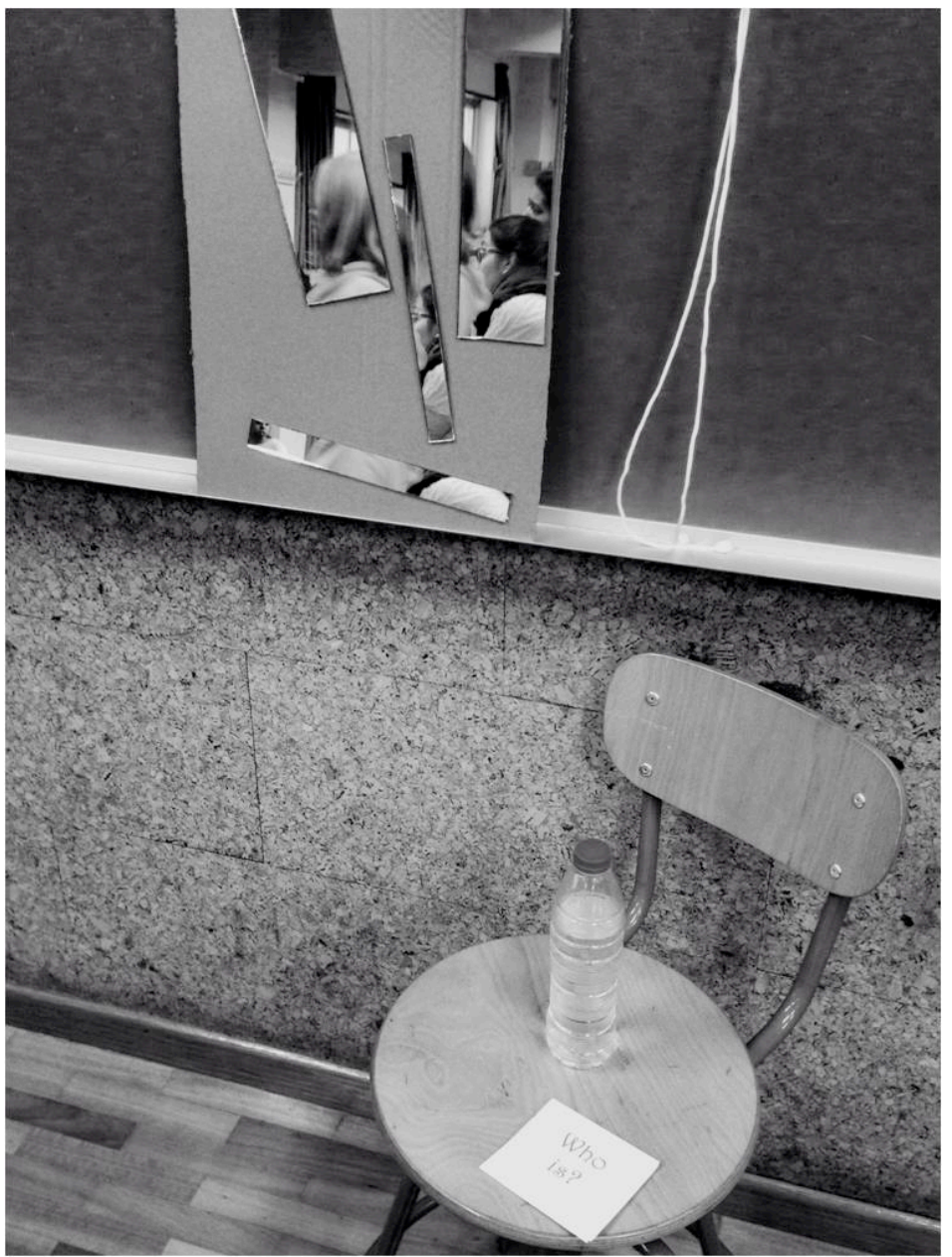

Figura 2: Alice Bajardi (2015). Portraits. Digital photo.

\section{Referencias}

Abad, J. (2012): "Usos y funciones de las artes en la educación y el desarrollo humano". En Jiménez. Lucina et al. Educación artística, cultura y ciudadanía. Colección Metas Educativas. Madrid: OEI-Fundación Santillana.

Bajardi, Alice y Álvarez-Rodríguez, D. (2012): "Art education to develop creativity and critical skills in digital society: integrating the tradition in an e-learning environment." Communications of the International Conference The Future of Education Conference Proceedings.

Bajardi, Alice y Álvarez-Rodríguez, D. (2014). Contribuciones de la educación artística a la construcción de la identidad profesional docente: competencias básicas y comunicativas. Historia y Comunicación Social, 18, 615-626.

Dewey, J. (2007): Como pensamos. Barcelona: Paidós.

Efland, A. (2004): Arte y cognición. La integración de las artes visuales en el currriculum. Barcelona: Octaedro.

Eisner, E. W. (1995): "La incomprendida función de las artes en el desarrollo humano". Revista Espeñola de Pedagogía, vol. 50, nº 191.

Lobato Fraile, C. (1997): "Hacia una comprensión del aprendizaje cooperativo”. Revista de Psicodidáctica, nº 4, 39-76.

Palacios, A. (2009): "El arte comunitario: origen y evolución de las prácticas artísticas colaborativas". Papeles de arteterapia y educación artística para la inclusión social. Vol 4, 197-211.

Read, H. (2007): Educación por el arte. Barcelona: Paidós. 